\title{
PERCEPÇÕES DE ADOLESCENTES BRASILEIROS E MEXICANOS SOBRE SEUS PAIS
}

\section{BRAZILIAN AND MEXICAN ADOLESCENTS'PERCEPTIONS ABOUT THEIR PARENTS}

\section{Celina Imaeulada Girardi 1}

GIRARDI, C. I. Percepções de Adolescentes Brasileiros e Mexicanos sobre seus Pais. Rev. Bras. Cresc. Desenv. Hum., São Paulo, 8 ( 1/2), 1998.

Resumo: Para se compreender os adolescentes, é necessário analisar como julgam a relação que mantêm com os seus pais, como é e como está no presente, e como foi, em muitas ocasiões, no passado. Ou seja, é preciso analisar como os adolescentes descrevem o grau e a forma com que estabelecem diferenças entre os progenitores quanto a diversos aspectos como: afeto, amizade, independência, igualdade, dominação, etc. Muitas vezes as diferenças, em termos das percepções dos filhos sobre seus pais, fundamentam-se na variação cultural entre os grupos ou nos conflitos entre as gerações mais jovens e as mais adultas, devido ao processo de formação de identidade que enfrenta todo adolescente. Por estas razões, o presente trabalho procura analisar como os adolescentes de duas culturas diferentes (brasileira e mexicana) descrevem seus pais, tomando como base um conjunto de adjetivos apresentados através de um Diferencial Semântico. Os resultados desta investigação (semelhanças e diferenças) constituem a contribuição do presente artigo.

Palavras-chave: adolescentes brasileiros e mexicanos; descritores de pai e mãe; diferencial semântico.

\section{INTRODUÇÂO}

Algumas áreas da psicologia apresentam significativas contribuições ao conhecimento e à compreensão do ser humano. A Psicologia Clínica considera que, para poder entender as pessoas, é necessário o conhecimento de seu passado. A Psicologia Social e a Psicologia do Desenvolvimento pesquisam como as pessoas se auto avaliam c, ou como avaliam suas vidas, com base em suas próprias opiniões ou conceitos sobre seu passado (PIPP,.TENNINGS, SHAVER, LAMBORN \& FISHER, 1983). Estes autores relatam que uma reconstrução cognitiva e afetiva do passado constitui para a pessoa uma parte de sua adaptação ao presente.

Deste modo, uma das melhores maneiras de compreender os adolescentes consiste em analisar como julgam a relação que mantêm com seus pais, como se apresenta esta relação no presente e como foi, em muitas ocasiões, no passado. É igualmente importante conhecer como descrevem a relação com seus pais, ou seja, o grau e a forma com que estabelecem diferenças emocionais entre o pai e a mãe, em termos de dimensões aflitivas tais como: amor, independência, amizade e do-

1 Centro de Investigación da Universidad Intercontimental, México, D.F.

End.: Calle Patricio Sanz, 408 - Depto. 401, Col Del Valle 03100 - México, D.F. E-mail: celinagirardi@yahoo.com 
minação (BACK \& BOURQUE, 1970, cit. lo ROSENTHAL, DEMETRIOU \& EFKLIDES, 1989).

Os adolescentes, muitas vezes, caracterizain de maneira distinta a relação que estabelecein com cada um dos seus progenitores, descrevendo a relação com o pai como mais formal e distante, enquanto que com a mãe seria Dai s próxima, calorosa (SAFA, 1974), marcada por maior igualdade, amizade e também mais negociável do que a relação coto o pai (PIPP et al., 1983).

Vários autores pesquisaram a influência da cultura sobre a atitude dos pais frente aos filhos, os conflitos entre as gerações, ou a percepção dos filhos sobre os pais (MURILLO, 1976, POTAMIANOU, 1978, cit. in ROSENTHAL, DEMETRIOU \& EFKLIDES, 1989). Estes autores concluíram que as diferenças em termos de conflitos entre as gerações, assim como as referentes à resolução dos mesmos, podem apoiar-se na variação cultural entre os grupos.

De acordo com LUMMERTZ \& BIAGGIO (1987), na adolescência, o indivíduo precisa estabelecer uma identidade própria, fora de sua família, pois seus horizontes aumentam enquanto diminuem seus interesses centrados exclusivamente na família, e é iniciada concomitantemente a assimilação dos julgamentos e dos valores do seu próprio grupo o que, muitas vezes, gera dificuldades na relação com os pais em função da autoridade dos mesmos. Além disso, na sociedade contemporânea, os jovens buscam em grau inuito maior sua identidade independentemente da t'amília o que provoca freqüentes contlitos entre as gerações.

Considerando que as diferentes culturas podein influir na percepção que as novas gerações têm sobre seus pais, o presente estudo foi realizado com a finalidade de conhecer como os adolescentes mexicanos e brasileiros percebem e descrevem os seus progenitores.

\section{METODOLOGIA DE INVESTIGAÇÃO}

Participaram deste estudo 1422 adolescentes mexicanos ( $50 \%$ do sexo masculino e $50 \%$ do sexo feminino) e 810 brasileiros ( $45 \%$ do sexo masculino e $55 \%$ do sexo feminino), cujas idades estavam compreendidas entre os 14 e 19 anos. Todos os adolescentes eram estudantes do Segundo Grau. Em ambos países, os grupos forain equiparados quanto à porcentagem de alunos para cada uma das três séries do Segundo Grau e quanto à variável idade dos alunos.

A amostra mexicana foi constituída por estudantes das escolas Preparatórias da Universidad Nacional Autónoma do México, residentes no Distrito Federal. A amostra brasileira foi composta por estudantes das Escolas Públicas e Particulares de duas capitais do Sul do País, Florianópolis, capital do estado de Santa Catarina e Porto Alegre, capital do Rio Grande do Sul.

A atitude dos pais em relação aos filhos foi definida como "a percepção que os adolescentes têm sobre a maneira como foram tratados regularmente pelo pai e pela mãe" (GIRARDI, 1988, p. 154).

Para avaliar a atitude dos pais em relação aos filhos foram selecionados nove adjetivos bipolares que descrevem as maneiras de proceder, democráticas ou autoritárias, que os pais normalmente adotam com seus filhos. $\mathrm{O}$ tipo de instrumento utilizado foi o Difèrencial Semântico de Osgood, com os seguintes adjetivos bipolares: impositivo, acessível, amigável, responsável, compreensivo, exigente, dominante, consistente e rígido. As pontuações para qualificar os pais variavam de I a 5, ou seja, de muito a nada, para cada um dos adjetivos indicados. Os mesmos adjetivos foram utilizados para avaliar (separadamente) a atitude do pai e da mãe.

A escala para avaliar a atitude dos pais continha as instruções necessárias e foi aplicada aos adolescentes nas próprias salas de aula, durante as atividades regulares do período escolar.

\section{RESULTADOS}

Os dados obtidos no México e no Brasil foram analisados separadamente, para poder comparar os resultados destas duas culturas. Em primeiro lugar, foram realizadas as análises psicométricas requeridas pela investigação científica (GIRARDI, 1994)². Posteriormente, foram efetuadas as comparações entre os resultados de ambos países (GIRARDI, 1998).

2 As análises psicométricas realizadas foram as seguintes: análise de frequência para observar a distribuição das frequências da amostra total e a distribuição das opções de respostas dos itens da escala; prova t de Student para conhecer o poder de discriminação dos itens do instrumento (Diferencial Semàntico); Alpha de Cronbach para obter os coeficientes de consistêneia interina das escalas; Correlação Produto-momento de Pearson para analisar as correlações entre as escalas de pai e mãe. Estas análises psicométricas foram omitidas do presente artigo por já terem sido publicadas anterionnente (GIRARDI, 1994). 
Quadro 1 - Resultados da Escala Pai: Comparação entre México e Brasil

\begin{tabular}{|cc|}
\hline \multicolumn{2}{|c|}{ Escala Pai Democrático: semelhanças e diferenças } \\
Adjetivos obtidos no México & Adjetivos obtidos no Brasil \\
& Acessível \\
Acessível & Amigável \\
Amigável & Compreensivo \\
Compreensivo & Responsável \\
Responsável & \\
\hline & Escala Pai Autoritário: semelhanças e diferenças \\
Adjetivos obtidos no México & Adjetivos obtidos no Brasil \\
Impositivo & Impositivo \\
Exigente & Exigente \\
Dominante & Dominante \\
Rígido & Rígido \\
-- & Consistente \\
\hline
\end{tabular}

Quadro 2 - Resultados da Escala Mãe: Comparação entre México e Brasil

\begin{tabular}{|cc|}
\hline \multicolumn{2}{|c|}{ Escala Mãe Democrática: semelhanças e diferenças } \\
Adjetivos obtidos no México & Adjetivos obtidos no Brasil \\
Acessível & Acessível \\
Amigável & Amigável \\
Compreensiva & Compreensiva \\
Responsável & -- \\
\hline \multicolumn{2}{|c|}{ Escala Mãe Autoritária: semelhanças e diferenças } \\
\hline Adjetivos obtidos no México & Adjetivos obtidos no Brasil \\
Impositiva & Impositiva \\
Exigente & Exigente \\
Dominante & Dominante \\
Rígida & Rígida \\
-- & Consistente \\
\hline
\end{tabular}


Os dados dos quadros 1 e 2 (vide pág. anterior), foram selecionados por referirem-se às semelhanças e diferenças encontradas nos itens respondidos por todos os adolescentes mexicanos (1422) e por todos os adolescentes brasileiros (810).

De acordo com os resultados dos Quadros I e 2 (vide pág. anterior), tanto no México como no Brasil, uma parte dos adolescentes consideram os pais democráticos, e outra parte os percebe como autoritários. Considerando que as porcentagens das variáveis sociodemográficas sexo, idade e escolaridade foram bastante parecidas, tanto intra como inter países, omitiremos estes dados estatistícos no presente relato de pesquisa.

Quanto às semelhanças encontradas nas duas culturas, é possível observar que os adolescentes de ambos países consideram que o pai e a mae democráticos são acessíveis, amigáveis e compreensivos na relação que estabelecem com os filhos; enquanto que os pais autoritários são percebidos como impositivos, exigentes, dominantes e rígidos.

Com respeito às diferenças registradas, é interessante observar que nas escalas de pai e mãe democráticos, somente nos dados do México aparece o adjetivo responsável; enquanto que nas escalas de pai e mãe autoritários somente nos resultados do Brasil se observa a presença do adjetivo consistente.

\section{DISCUSSÃO}

Os adolescentes, ao responder ao Diferencial Semântico que lhes foi apresentado, tiveram de pensar em qual foi a atitude dos pais em relação a eles, desde a infância até o momento presente. Deste modo, a resposta individual que davam ao instrumento refletia como seus progenitores eram percebidos por eles, pois os resultados obtidos descreviam como cada sujeito percebia os próprios pais.

Os adjetivos da escala democrática, tanto no caso do pai como da mãe, trazem uma carga afetiva bastante expressiva, pois além de serem acessíveis e compreensivos, os pais são também amigos, ou seja, companheiros, o que os caracteriza como bastante positivos. Isto não ocorre com os pais classificados como autoritários, pois são denotados por adjetivos onde se percebe uma carga bastante negativa.

A carga afetiva presente na escala democrática, tanto nos dados do Brasil como nos do México, encontra apoio nos estudos de TRIANDIS, LISANSKY, MARIN \& BETANCOURT (1984) em que relatam que hispanos e latino americanos apresentam uma característica cultural e um padrão de interação social bastante particulares refletidos na 'simpatia'. A pessoa que mani- festa esta qualidade pessoal é percebida como agradável e de fácil convivência, mostrando certo nível de conformidade e de habilidade para compartilhar seus sentimentos com os outros, comportando-se com dignidade e respeito em relação aos demais e parecendo esforçar-se para manter a harmonia nas relações interpessoais.

Segundo DÍAZ-CARABANO (1974) e DIAZ-GUERRERO (1972, 1985), o afeto constitui um valor muito importante para a cultura mexicana. Este valor foi também encontrado por PINTO \& FTNGER (1989), em um estudo realizado com estudantes sul-americanos (brasileiros e chilenos), os quais outorgaram grande importância ao 'afeto' e à 'responsabilidade', como determinantes fundamentais dos modos de conduta que têm um foco interpessoal.

Esta qualidade pessoal dos latinos foi confirmada no presente estudo pelos adolescentes mexicanos e brasileiros, os quais reconhecem que seus pais são abertos e tolerantes (acessíveis) e, ao mesmo tempo, carinhosos e dedicados (amigáveis e compreensivos), adjetivos que estão presentes na escala democrática.

No entanto, o profundo respeito e a obediência ilimitada que os filhos devem demonstrar aos pais fazem com que, às vezes, eles também os percebam como autoritários, ou seja: impositivos, exigentes, dominantes e rígidos (o que caracteriza a escala de autoritarismo do presente estudo). Este dado concorda com a descrição de MURILLO (1976) de que os pais méxico-americanos são relativamente autoritários, mais reservados e exigem mais respeito com a criança uma vez esta atingindo a puberdade. Outros autores acrescentam que os pais são geralmente mais permissivos com o filho ainda criança do que na puberdade ou na adolescência (TRIANDIS, MARIN, HUI, LISANSKI \& OTTATI, 1984).

Essas mudanças na atitude ou no tratamento dos pais com os filhos nesta fase podem ter razões sociais, culturais ou ser resultante da aprendizagem adquirida na interação com o ambiente. Por outro lado, a necessidade do próprio adolescente de buscar sua identidade fora da família, conforme mencionam LUMMERTZ \& BIAGGIO (1987), provoca constantes conflitos entre as gerações e faz com que os jovens percebam os seus pais como autoritários.

No México, a pesquisa foi realizada no Distrito Federal, para onde imigram gente de diferentes regiões do pais; portanto, é provável que os dados obtidos sejam representativos da cultura mexicana. No Brasil, o instrumento foi aplicado no Sul do país, aonde se verifica a presença de uma cultura marcadamente européia, com influência particularmente italiana, alemã e portuguesa. Por esta razão, é recomendável ter cautela nas 
implicações dos resultados obtidos, evitando generalizações para todo o território brasileiro.

Ainda que as diferenças quanto à seleção da amostra dos dois países possam constituir uma explicação plausível para os resultados apresentados, dado que certos aspectos culturais (éticos, religiosos, arnbientais, educacionais, etc.) podem acentuar as diferenças culturais intra e entre os países, será necessário prosseguir os estudos para tentar encontrar explicações mais claras para os resultados da presente pesquisa.

Por esta razão, a próxima etapa consistirá em buscar explicações teórico-metodológicas mais profundas para as diferenças quanto aos adjetivos: responsável e consistente (presentes nos dados de México e Brasil, respectivamente), para poder entender o significado semântico destes adjetivos nas duas culturas contempladas.

\begin{abstract}
In order to understand adolescents, it is necessary to analyse how they judge the relation they have with their parents, how this relation is in the present, and how it was, in many occasions, in the past. In other words, it is necessary to analyse how the adolescents describe the form and the extent to which they establish dif'ferences be^hveen the parents with respect to many aspects such as: affection, friendship, independence, equality, domination, etc. Frequently, the differences regarding children's perceptions about heir parents are based on the cultural variation among the groups or on the confficts between younger and older generations, due to the process of identity formation undergone by every adolescent. For these reasons, the present paper tries to analyse how adolescents from hvo distinct cultures (Brazilian and Mexican) desc ibe their parents, based on a set of adjectives presented by means of a Semantic Diff'erential. The results of this investigation (similarities and differences) constitute the contribution of'the present article.
\end{abstract}

Key-words: Brazilian and Mexican adolescents; desc^libers of'father a ^^ld n $\sim$ '’ner; semantic differential.

\section{REFERÊNCIAS BIBLIOGRÁFICAS}

DÍAZ-CARABAÑO, M. Estudio caractereológico en un grupo de familias mexicanas. México, 1974. [Tese de Doutorado - Universidad Nacional Autónoma de México].

DÍAZ-GUERRERO, R. Hacia una teoria histórica-biopsico-socio-cultural del comportamiento humano. México, Trillas, 1972.

DÍAZ-GUERRERO, R. Psicologia del Mexicano. México, Trillas, 1985.

GIRARDI, C. I. Un modelo de incapacidad aprendida para adolescentes mexicanos. México, 1988. [Tese de Doutorado - Universidad Nacional Autõnoma de México].

GIRARDI, C. I. Actitud hacia los padres en México y en Brasil. La Psicologia Social en México. México, Asociación Mexicana de Psicología Social, V: 693-699, 1994.

GIRARDI, C. I. Attitude towards parents in México and Brasil. In: XIV International Congress for Cross-Cultural Psychology. Washington University, Bellingham, U.S.A., 1998, p. 95.

LUMMERTZ, J.G.; BIAGGIO, A. M. Desenvolvimento de uma escala para medir o nível de satisfação do adolescente em sua família; Psicologia: Teoria ePesquisa, 3(2): 124-137, 1987.

MURILLO, N. The Mexican-American family. In: HERNANDEZ, C. A.; HAUG, M. J.; WAGNER, N. N. (eds.). Chicanos: Social and psychological perspectives. St. Louis, Mosby, 1976, p. 1 5-25.
NUNNALLY, J. Psychometric theory. New York, McGraw Hill, 1987.

OSGOOD, C. E.; SUCI, J. G; TANNENBAUM, P. H. The measurement of meaning. Urbana, University of Illinois Press, 1957.

PINTO, R. F. M; FINGER, A. P. Valores termina-les e instrumentales de estudiantes universita-rios chilenos y brasileños: un estudio explora-torio. Arquivos Brasileiros de Psicologia, 4(2): 2933, 1989.

PIPP, S.; JENNINGS, S.; SHAVER, P.; LAMBORN, S.; FISHER, K. W. Adolescents theories about the development of their rela-tionships with parents. Journal of Personality and Social Psychology, 48(34): 991-1001, 1983.

ROSENTHAL, A.; DEMETRIOU, A.; EFKLIDES, A. Across-national study of the influence of culture on conflict between parents and adolescents. International Journal of Behavioral Development, 12(2): 207-219, 1989.

ROSENTHAL, D. A. Intergenerational conflict and cultural study of immigrant and nonimmigrant adolescents and their parents. Genetic Psychology Monographs, 109: 53-75, 1984.

SAFA, H.I. The urban poor of Puerto Rico. A study in development and inequality. New York: Holt, Rinehart \& Winston, 1974.

TRIANDIS, H.C.; LISANSKI, J.; MARÍN, G.; BETANCOURT, H. Simpatia as a cultural script of Hispanics. Journal of Personality and Social Psychology, 47(ó): 1363-1375,1984.

TRIANDIS, H.C.; MARIN, G.; HUI, C.H.; LISANSKY; J.; OTTATI, V. Role perceptions of Hispanic young adults. Journal of Crosscultural Psychology, 15(3): 297-220, 1984. 